\title{
Implicit memory, the serial position effect, and test awareness
}

\author{
JOHN M. RYBASH and JOYCE L. OSBORNE \\ Hamilton College, Clinton, New York
}

\begin{abstract}
Separate groups of college students ( $n=20$ per group) were administered either an explicit memory task: (free recall, FR, or cued recall, CR) or an implicit memory task (word-stem completion, WSC) for a list of previously studied words. Primacy and recency effects were observed for the subjects performing the FR task. A recency effect, but not a primacy effect, was displayed for the subjects performing the CR and WSC tasks. Within the WSC condition, the test-aware subjects displayed a greater amount of priming than did the test-unaware subjects. Furthermore, the test-aware subjects in the WSC condition displayed a smaller amount of priming than the subjects in the CR condition.
\end{abstract}

Research on the topic of implicit memory has become increasingly popular among cognitivists and neuropsychologists (for reviews, see Roediger, 1990; Schacter, 1987). A memory task is implicit to the extent that it does not require a subject to intentionally or deliberately remember a past experience. Implicit memory has been assessed by a wide range of tasks, such as repetition priming (e.g., word-stem completion) and perceptual identification. Implicit memory tasks may be contrasted with explicit memory tasks. Tasks of the latter type require that a subject intentionally recollects a previous event or episode. Unlike implicit memory, it is accompanied by the phenomenological quality of "pastness" (Schacter, 1987). Most of the traditional tests of memory used in psychological research (e.g., recognition, cued recall, free recall) may be regarded as measures of explicit memory.

\section{Serial Position Effect}

One hallmark of explicit memory is the serial position effect-enhanced memory for information at the beginning (the primacy effect) and the end (the recency effect) of a word list relative to the middle of the list. Explanations of the serial position effect have generally taken two different forms. Dual-memory theorists (e.g., Atkinson \& Shiffrin, 1977) maintain that the primacy effect reflects the retrieval of information that has been transferred from short-term memory (STM) to long-term memory (LTM), whereas the recency effect entails retrieval of information from STM. Alternatively, it has been reported (see Fitzgerald, 1988; Pillemer, Goldsmith, Panter, \& White, 1988) that the major components of the serial position effect may be observed for information that must be stored in LTM (e.g. , having more vivid memories of the beginning and/or end of one's freshman year of college). It may be the case that individuals consciously/intentionally use

Correspondence should be addressed to John M. Rybash, Department of Psychology, Hamilton College, Clinton, NY 13323. the beginning and end of a time segment as "anchors" or "benchmarks" from which to search LTM.

Both of the above-mentioned theoretical explanations lead to the hypothesis that the serial position effect should not be displayed when memory for a previously presented word list is tested implicitly. This hypothesis is based on the premises that (1) the STM-LTM distinction applies to explicit memory but not implicit memory (e.g., Squire, 1987; Tulving \& Schacter, 1990), and (2) tasks of implicit memory do not involve the implementation of conscious/ intentional retrieval strategies (Schacter, 1987). Thus, one purpose of the present study was to test the hypothesis that the serial position effect would be observed when memory for a previously studied word list was assessed by two different explicit memory tasks-free recall (FR) and cued recall (CR)-but the serial position effect would not be observed when memory for a previously studied word list was assessed by an implicit memory task-wordstem completion (WSC).

The serial position effect is typically observed on FR tasks. We chose to include a CR condition in the present study for three reasons. First, little is known about whether the serial position effect is displayed on CR tasks. Second, we reasoned that it is more meaningful to compare performance on a WSC task with performance on a CR task than with an FR task. This is because, although the instructions given to subjects in CR and WSC tasks are different, the stimuli employed in these tasks are identical. Third, if the serial position effect is displayed on the FR task but not on the CR and WSC tasks, it would add evidence to the claim that certain memory phenomenon (e.g., the serial position effect) are unique to specific tasks (e.g., FR) within a specific memory domain (e.g., explicit memory).

\section{Test Awareness}

Another purpose of the present study was to examine the issue of test awareness in implicit memory (see Schacter, Bowers, \& Booker, 1989). Test awareness refers to a sub- 
ject's beliefs that the stimulus materials he/she encounters on an implicit memory task correspond to previously studied items. It has long been recognized that amnesics remain test-unaware during the completion of an implicit memory task (Squire, 1987; Schacter, 1990). Whether or not normal (i.e., nonneurologically impaired) subjects remain test-unaware is an extremely important issue. For example, Schacter et al. (1989) maintained that test-aware subjects may adopt a variety of conscious/intentional retrieval strategies. Thus, their performance may reflect a type of involuntary explicit memory rather than genuine implicit memory (Schacter et al., 1989).

In a recent experiment, Bowers and Schacter (1990) reported that one half of their sample of college students remained test-unaware while performing a WSC task, and that test-aware subjects completed just as many word stems as did test-unaware subjects. We sought to replicate these results. Furthermore, we wanted to test the hypothesis that a serial position effect would manifest itself for test-aware subjects, but not test-unaware subjects, in the WSC condition. This hypothesis is based on the assumption that test-aware subjects, but not test-unaware subjects, would adopt explicit retrieval strategies similar to those used by subjects in the CR and FR conditions.

\section{Method}

Subjects. Sixty college students enrolled in introductory psychology courses served as subjects $(M=22.5$ years of age). All subjects were native English speakers and did not suffer from any neurological illness or handicap.

Materials. Stimuli for the explicit and implicit memory tasks consisted of $\mathbf{4 8}$ words that were selected from the pool developed by Kucera and Francis (1967). Each word was five to seven letters long, had a unique three-letter stem, had a minimum of four alternative stem completions, and was never the most frequent completion of its stem.

Twenty-four of the aforementioned words were selected at random to be used as the stimuli during the study phase of the present experiment. Each of these words was typed separately on a $14 \times 22 \mathrm{~cm}$ sheet of white paper. Below each word was a Likert scale ranging from 1 to 7 on which the subjects were instructed to rate the pleasantness of the word $(1=$ very unpleasant, $7=$ very pleasant $)$. All of the words were contained in a 24-page booklet.

Separate tests of FR, CR, and WSC were constructed. The FR task consisted of two columns of 4-cm lines consecutively numbered 1 through 24 , printed on a standard $28 \times 22 \mathrm{~cm}$ sheet of white paper. The CR and WSC tasks consisted of three columns of 16 three-letter word stems, printed on a standard $28 \times 22 \mathrm{~cm}$ sheet of white paper. One half of the word stems consisted of all 24 of the words that were previously rated for pleasantness. The other half of the word stems (24) consisted of the remaining words from the master list that were not rated for pleasantness. For example, the word APPEAL, which was contained on the study list, appeared as APP_ on the CR and WSC tasks. An equal number (eight) of three-letter stems for previously studied words were distributed randomly within each of the three columns.

An awareness questionnaire modeled after the one used by Bowers and Schacter (1990) was also constructed. This questionnaire contained three items as follows: (1) What do you think was the general purpose of the word-stem completion test that you just finished? (2) What was the general strategy you used in completing the word stems? (3) While doing the word-stem completion test, did you notice whether you completed some of the stems with words you rated for pleasantness in the earlier word pleasantness task? The subjects who referred to the words they rated for pleasantness during the study phase in response to Questions 1 and 2 or who responded affirmatively to Question 3 were classified as test-aware. The subjects who did not mention the words en- countered during the study phase in response to Questions 1 and 2 and who responded negatively to Question 3 were classified as test-unaware.

Procedure. The subjects were randomly assigned to one of three different groups: FR, CR, and WSC ( $n=20$ per group). The subjects in all groups were told the purpose of the study was to measure the verbal abilities of college students. After filling out a demographic questionnaire, all subjects completed two filler tasks: the vocabulary subtest of the WAIS and an orthographic differentiation task (Hunt \& Toth, 1990). Next, all subjects rated the pleasantness of the 24 previously described words. The subjects were allowed approximately $3 \mathrm{sec}$ to say each word aloud and circle the appropriate pleasantness rating for that word.

Immediately after the termination of the pleasantness task, the subjects in the FR condition were asked to write down all of the words they could remember that they had just rated the pleasantness of. The subjects in the CR condition were shown $\mathbf{4 8}$ word stems and were told to complete only the stems that would spell words they had just rated the pleasantness of. It was emphasized that they should not complete any word stems with words that they had not rated for pleasantness on the previous list. The subjects in the WSC condition were presented with the same word stems as those employed in the CR condition and were instructed to complete as many word stems as possible with the first letters that came to mind that spelled a valid English word (except proper nouns). Immediately following the WSC task, all subjects in the WSC condition were administered the awareness questionnaire.

Design. A 3×6 mixed design was employed. Memory task (FR, CR, WSC) was the between-subject factor. The within-subject factor was word position of the 24 words that were rated for pleasantness. Specifically, the 24 words were broken down into six positions: Position $1=$ Words 1-4, Position 2 = Words 5-8; etc. The dependent variable was the proportion of words remembered by the subjects in each memory task condition at each of the six different word list positions.

\section{Results}

Overall analysis. The data were analyzed by a 3 (memory task) $\times 6$ (word position) mixed ANOVA. Results showed main effects for memory task $[F(2,57)=24.09$, $p<.001]$ and word position $[F(5,285)=8.95, p<$ $.0001]$ and a significant memory task $\times$ word position interaction $[F(10,285)=2.12, p<.023]$.

Post hoc analysis (note that all post hoc comparisons involved the use of the Fischer $t$ statistic at $p<.05$ ) of the memory task $\times$ word position interaction (see Figure 1) indicated that, in the FR condition, the subjects recalled more words in Position 1 than in Position 2, an equal number of words in Positions 2, 3, 4, and 5, and significantly more words in Position 6 than in Position 5. Thus, the subjects in the FR condition demonstrated a classic special position effect-a primacy effect for words that occupied Position 1 and a recency effect for words that occupied Position 6. The subjects in the CR condition completed just as many stems for words in Positions 1 , 2,3 , and 4 . Their performance declined for words in Position 5, relative to Position 4; however, it improved for words in Position 6, relative to Position 5. Thus, they demonstrated a recency effect, but not a primacy effect. The subjects in the WSC condition completed just as many stems for words in Positions 1 and 2, marginally more stems $(p<.06)$ for words in Position 2 than for words in Positions 3 and 4, less stems for words in Position 5 than for words in Position 4, and more stems for words in Position 6 than for words in Position 5. These subjects, just as those in the CR condition, displayed a recency effect. Unlike the subjects in the CR condition, they seemed to exhibit a marginally significant primacy effect. 


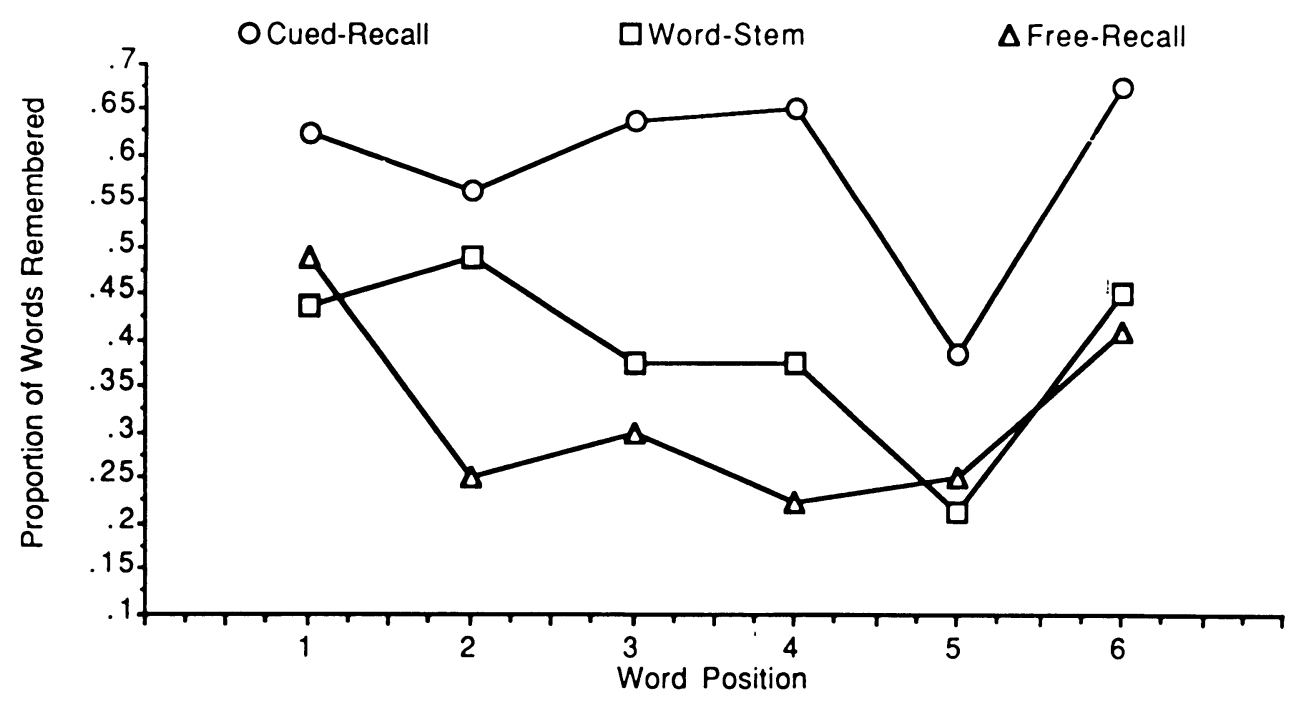

Figure 1. Proportion of words remembered as a function of word position and memory task condition.

To make a more direct comparison of the performance of the subjects in the FR, CR, and WSC conditions across all six word positions, three separate $2 \times 6$ mixed ANOVAs were also conducted. These ANOVAs examined the performance of the subjects in the FR versus $C R$ conditions, the $F R$ versus WSC conditions, and the CR versus WSC conditions. All of the ANOVAs revealed main effects for memory task and word position. The first two of these ANOVAs also yielded significant memory task $x$ word position interactions ( $p<.028$, in both instances). The third ANOVA, which compared the performance of the subjects in the CR and WSC conditions, failed to produce a significant memory task $\times$ word position interaction $(p<.332)$. Thus, these analyses strengthened the conclusion that the FR group displayed both a primacy and a recency effect, while the CR and WSC groups displayed a recency effect but not a primacy effect.

Finally, a comparison of the proportion of word stems completed for previously studied words $(M=.393)$ versus nonstudied words $(M=.075)$ indicated a significant amount of priming for the subjects in the WSC condition $[t(19)=10.85, p<.0001]$.

Analysis of the performance of test-aware versus testunaware subjects. Of the 20 subjects in the WSC condition, 15 were found to be test-aware and 5 were found to be test-unaware. A 2 (awareness status: aware vs. unaware) $\times 6$ (word position) mixed ANOVA revealed main effects for awareness status $[F(1,18)=6.27, p<.022]$ and word position $[F(5,90)=4.28, p<.001]$. The awareness status $X$ word position interaction (see Figure 2) proved to be insignificant $[F(5,90)=0.28, p<.93]$.

Comparisons were made regarding the proportion of studied versus nonstudied word stems that were completed by the test-aware versus the test-unaware subjects. These analyses revealed a significant amount of priming for the subjects in both awareness conditions. Specifically, the test-aware subjects completed more stems $[t(14)=10.43$, $p<.0001]$ for studied $(M=.422)$ than for nonstudied words $(M=.07)$. The test-unaware subjects completed more stems $[t(4)=6.31, p<.002]$ for studied $(M=$ $.292)$ than for nonstudied words $(M=.07)$.

We also conducted a $3 \times 6$ mixed ANOVA that contrasted the performance of the subjects in the CR condition with that of the test-aware and test-unaware subjects in the WSC condition. Main effects were revealed for group $[F(2,37)=13.21, p<.0001]$ and word position $[F(5,185)=7.70, p<.0001]$. Analysis of the main effect for group indicated that the subjects in the CR condition completed more stems $(M=.590)$ than did the test-aware subjects in the WSC condition $(M=.422)$; the test-aware subjects in the WSC condition completed more stems than did the test-unaware subjects in the WSC condition $(M=$ .292). The group $\times$ word position interaction was nonsignificant $[F(10,185)=0.70, p<.726]$.

\section{Discussion}

The present study yielded two important findings. First, relative to the manifestation of a serial position effect, it was discovered that the subjects in the FR condition performed in a manner that was different from the subjects in the CR and WSC conditions. The subjects in the FR condition displayed both primacy and recency effects. The subjects in the CR and WSC conditions demonstrated a recency effect but not a primacy effect. These results suggest that the pattern of performance exhibited by the subjects across all six word positions was driven by the demands of a particular memory task independent of whether the task was classified as implicit or explicit. These results suggest that, in some instances, tasks within the domain of explicit memory (i.e., FR vs. CR) may yield dramatically different patterns of performance, and tasks representative of different memory domains (CR vs. WSC) may produce similar outcomes (cf. Tulving \& Schacter, 1990).

Second, with regard to the issue of test awareness, it was discovered that (1) both the test-aware and the test-unaware subjects in the WSC condition exhibited significant amounts of priming; (2) the test-aware 


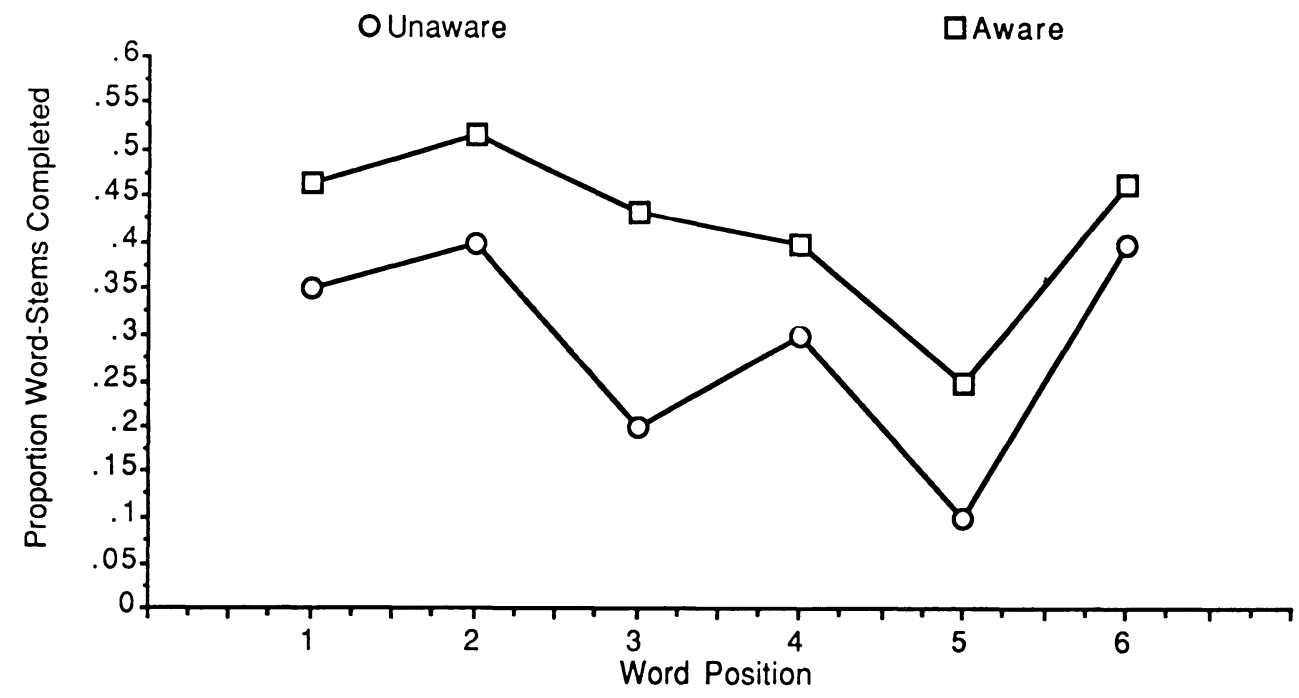

Figure 2. Proportion of word stems completed by the test-aware and test-unaware subjects in the WSC condition.

subjects in the WSC condition completed more word stems than did the test-unaware subjects in the WSC condition; (3) the subjects in the CR condition completed more word stems than did the test-aware subjects in the WSC condition; and (4) the subjects in the WSC condition, regardless of awareness status, displayed an identical pattern of performance across all six word list positions relative to the subjects in the CR condition. These findings have important implications for researchers examining various aspects of implicit memory via a WSC task. For example, it has been suggested (e.g., Howard, 1988) that test-aware subjects may use an explicit strategy while completing word stems and therefore inflate and contaminate their performance on a WSC task. Arguments such as these gain credibility when one considers that test-aware subjects exhibit a greater amount of priming than do test-unaware subjects.

The results of the present study regarding test awareness and implicit memory are inconsistent with some of the data obtained by Bowers and Schacter (1990). They reported equal numbers of test-aware versus testunaware subjects. We found a greater number of test-aware subjects than test-unaware subjects. Three different factors may have a bearing on this discrepancy. First, in the present study, the test phase in the WSC condition consisted of an equal number of targets (24) and distractors (24). Also, all 24 of the studied words were included as targets in the test list. The test list in the Bowers and Schacter (1990) study consisted of 12 targets and 63 distractors. The 12 targets were selected from 32 different words that appeared on a study list. Thus, given the ratio of targets to distractors in the test phases of both experiments, it would have been much easier for the subjects to notice previously studied words (and therefore be classified as test-aware) in the present study than in Bowers and Schacter's (1990) study. Second, in the present study, the WSC task was presented immediately after the subjects rated the original list of 24 words for pleasantness. Bowers and Schacter (1990) presented the WSC task to their subjects approximately $10 \mathrm{~min}$ after they studied a word list. Third, all of the subjects in the present investigation made semantic judgments about the words on the original study list. Perhaps the deeper levels of processing that are engendered by semantic encoding facilitate test awareness.

In conclusion, the results of the present experiment suggest that $C R$ and WSC tasks produce a similar pattern of performance with regard to subjects' ability to remember (implicitly or explicitly) words that occupied specific serial positions on a previously studied word list. It was found that the majority of the subjects in the WSC condition were testaware. These test-aware subjects completed significantly more word stems than did the test-unaware subjects; but they completed fewer word stems than did subjects in the CR condition.

\section{REFERENCES}

Atkinson, R. C., \& Shiffrin, R. M. (1977). Human memory: A proposed system and its control processes. In G. W. Bower (Ed.), $\mathrm{Hu}$ man memory: Basic processes. New York: Academic Press.

Bowers, J. S., \& SCHACTER, D. L. (1990). Implicit memory and test awareness. Journal of Experimental Psychology: Learning, Memory, \& Cognition, 16, 404-416.

Fitzgerald, J. M. (1988). Vivid memories and the reminiscence phenomenon. Human Development, 31, 260-270.

HowARD, D. (1988). Implicit and explicit assessment of cognitive aging. In M. L. Howe \& C. J. Brainerd (Eds.), Cognitive development in adulthood: Progress in cognitive development research (pp. 3-37). New York: Springer-Verlag.

Hunt, R. R., \& Toтн, J. P. (1990). Perceptual identification, fragment completion, and free recall: Concepts and data. Journal of Experimental Psychology: Learning, Memory, \& Cognition, 16, 282-290.

KuČera, H., \& Francis, W. N. (1967). Computational analysis of present-day American English. Providence, RI: Brown University Press.

Pillemer, D. B., Goldsmith, L. R., Panter, A. T., \& White, S. H. (1988). Very long-term memories of the first year in college. Journal of Experimental Psychology: Learning, Memory, \& Cognition, 14, 709-715.

ROEDIGER, H. L., III (1990). Implicit retention without remembering. American Psychologist, 45, 1043-1056.

SCHACTER, D. L. (1987). Implicit memory: History and current status. Journal of Experimental Psychology: Learning, Memory, \& Cognition, 13, 501-518.

SChaCter, D, L. (1990). Perceptual representation systems and implicit memory: Toward a resolution of the multiple memory systems debate. In A. Diamond (Ed.), Development and the neural bases of higher cognitive function (pp. 543-571). New York: Annals of the New York Academy of Sciences.

SCHACTER, D. L., BOWERS, J. S., \& BOOKER, J. (1989). Intention, awareness, and implicit memory: The retrieval intentional criterion. In S. Lewandowsky, J. Dunn, \& K. Kirsner (Eds.), Implicit memory: Theoretical issues (pp. 47-65). Hillsdale, NJ: Erlbaum.

SQUIRE, L. R. (1987). Memory and brain. New York: Oxford University Press.

Tulving, E., \& Schacter, D. L. (1990). Priming and human memory systems. Science, $247,301-306$. 